\title{
When Second Best Might Be the Best: Using Hospitalization Data to Monitor the Novel Coronavirus Pandemic
}

\author{
Peter J. Mallow ${ }^{1 *}$ and Michael Jones ${ }^{2}$ \\ ${ }^{1}$ Department of Health Services Administration, Xavier University, Cincinnati, OH, United States, ${ }^{2}$ Department of Economics, \\ University of Cincinnati, Cincinnati, $\mathrm{OH}$, United States
}

The novel coronavirus' high rate of asymptomatic transmission combined with a lack of testing kits call for a different approach to monitor its spread and severity. We proposed the use of hospitalizations and hospital utilization data to monitor the spread and severity. A proposed threshold of a declining 7-day moving average over a 14-day period, "7\&14" was set to communicate when a wave of the novel coronavirus may have passed. The state of Ohio was chosen to illustrate this threshold. While not the ideal solution for monitoring the spread of the epidemic, the proposed approach is an easy to implement framework accounting for limitations of the data inherent in the current epidemic. Hospital

OPEN ACCESS

Edited by:

Sandra C. Buttigieg

University of Malta, Malta

Reviewed by:

Guenka Ivanova Petrova, Medical University-Sofia, Bulgaria Brian Godman,

Karolinska Institutet (KI), Sweden

*Correspondence:

Peter J. Mallow

mallowp@xavier.edu

Specialty section:

This article was submitted to

Health Economics,

a section of the journal

Frontiers in Public Health

Received: 12 May 2020

Accepted: 22 June 2020

Published: 10 July 2020

Citation:

Mallow PJ and Jones M (2020) When Second Best Might Be the Best: Using Hospitalization Data to Monitor the Novel Coronavirus Pandemic.

Front. Public Health 8:348,

doi: 10.3389/fpubh.2020.00348 administrators and policy makers may benefit from incorporating this approach into their decision making.

Keywords: coronavirus, COVID-19, hospitals, Ohio, surveillance

\section{INTRODUCTION}

Before government officials relax stay-at-home orders and hospitals resume elective procedures, decision-makers must accurately estimate the trend, severity, and prevalence of the novel coronavirus in a geographic region. Ideally, public health agencies would conduct active surveillance of infections in the general population (1-3). The results from this first-best solution represent a coincident indicator of COVID-19's prevalence in a population. However, the fact that the novel coronavirus has a high rate of asymptomatic transmission hinders the usefulness of this approach $(4,5)$.

Further hindering the disease surveillance is the limited number of novel coronavirus test kits as of April, 2020. Many states like Ohio prioritize the individuals who are eligible for testing (6). Ohio and many other states recommend that all individuals who exhibit symptoms should be tested. However, hospitalized individuals and healthcare workers are given first priority. Individuals in long-term care and first responders are given a lower priority, and individuals in the general population have the lowest priority. While this prioritization redirects resources to their most effective use, the number of positive cases represents a biased sample of the general population. This tradeoff suggests that the number of positive test cases in a population does not necessarily reflect the actual prevalence of COVID-19 nor the infection rate trend.

As a lagging indicator, COVID-19 hospitalizations would normally be considered a second-best solution to measuring a trend in the infection rate. However, given the sample bias reflected in prioritized testing and asymptomatic transmission, we propose that COVID-19 hospitalizations combined with a capacity measure offer the best approach to measuring trends in COVID-19 
infections. COVID-19 deaths present an even longer lag time than hospitalizations, and so they are not viewed as suitable of a measure. We chose the state of Ohio to illustrate our approach.

\section{METHODS}

The state of Ohio is one of several states that releases daily hospitalization data (5). However, they do not release length of stay (LOS) data. A literature search was performed in PubMed and the CDC Coronavirus website to identify studies published in March and April 2020 for LOS (7). A patient weighted pooled analysis was conducted to estimate the median LOS. The historical occupancy rate was obtained from the Centers for Disease Control and Prevention (CDC) National Center for Health Statistics for 2016 (8). Hospital capacity was defined as the number of staffed hospital beds (9).

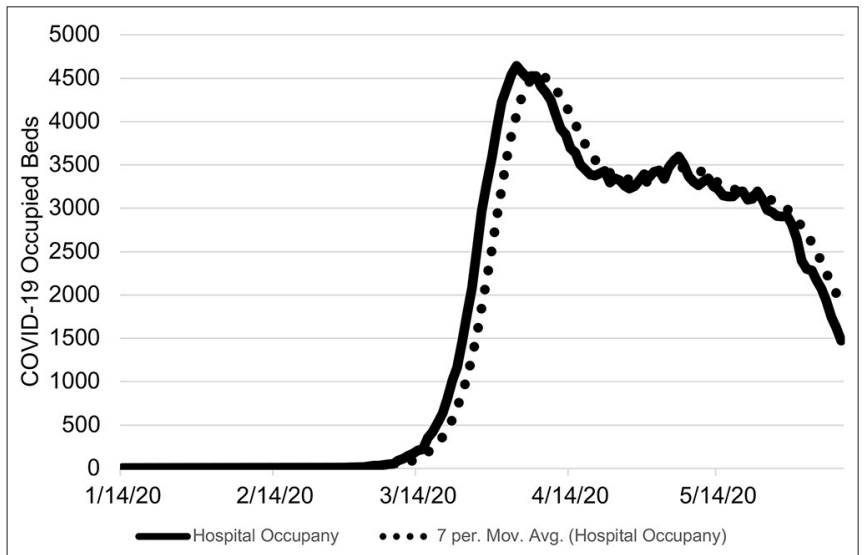

FIGURE 1 | Ohio COVID-19 Occupied Hospital Beds by Day. The number COVID-19 occupied hospital beds is shown from January 7 to June 8, 2020 with the 7-day moving average. The threshold of the proposed approach was met on April 30, 2020. On June 8, 2020, there were 1,472 estimated occupied beds due to COVID-19.

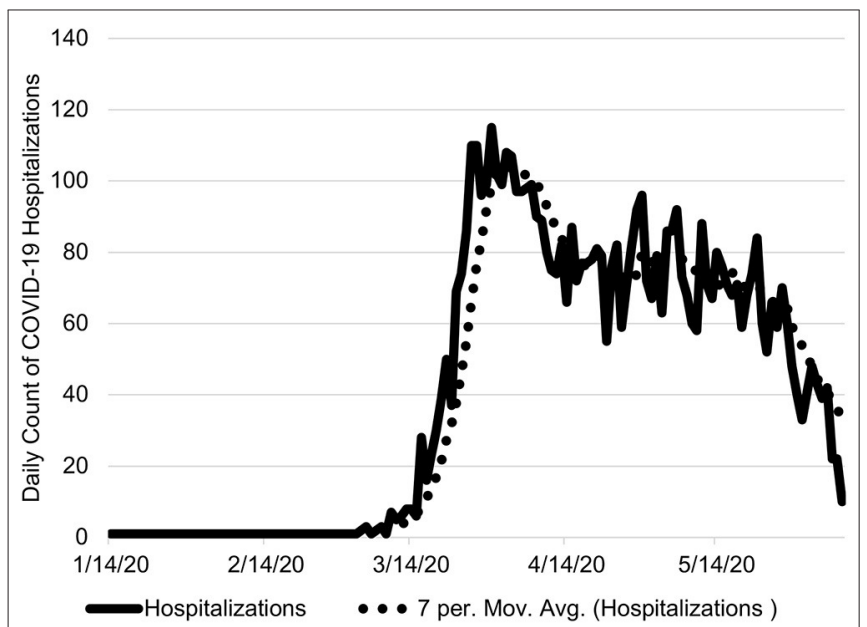

FIGURE 2 | Ohio Daily COVID-19 Hospitalizations. The number daily COVID-19 hospitalizations are shown from January 7 to June 8, 2020 with the 7-day moving average. On June 8, 2020, there were 10 COVID-19 hospitalizations.
The number of hospitalizations on a daily basis were multiplied by the median LOS to approximate the total number of bed days. Discharges based on LOS were subtracted to estimate a daily number of hospitalized COVID-19 patients. The number of occupied beds was calculated by multiplying the number of staffed beds by the pre-coronavirus occupancy rate. A 7day moving average was calculated by adding the number of hospitalized COVID-19 patients over each seven-day window and dividing by the time period. The threshold for assessing the passing of a novel coronavirus wave was set at a declining 7-day moving average over a 14-day period. The moving average period of 7-days was chosen to mitigate daily and weekend reporting effects and to be consistent with prior epidemiologic models (10-12). The length of time was chosen based on the current knowledge of the high end of the novel coronavirus incubation period (13). A further check included in the framework is stipulation that the 7 -day moving average plus the historical occupancy level did not exceed the number of staffed beds during this window. The research was conducted with de-identified publicly available data and is exempt from institutional review board review. All analysis was conducted in Microsoft Excel (Microsoft, Inc. Redmond, WA).

\section{RESULTS}

The application of this approach to the state of Ohio found the first wave of the novel coronavirus passed on April 30, 2020 (Figure 1). During the period of January 7 to June 8, 2020, there were 6,620 COVID-19 hospitalizations of which 624 did not have an associated admission date (Figure 2). Based on the median LOS of 4.9 days, these hospitalizations accounted for 24,265 hospital bed days (Table 1). The peak bed utilization based on the 7-day moving average occurred on April 9 with 4,642. At the peak, COVID-19 patients occupied $10 \%$ of the total staffed beds in Ohio. Combined with the occupancy rate, $\sim 73 \%(24,264)$ of staffed beds would have been in use on the peak day, remaining under capacity. The results were based on an imputed LOS and occupancy level for Ohio and were intended to illustrate this approach rather than inform decision making.

\section{DISCUSSION}

A critical component of monitoring the novel coronavirus pandemic is the availability of reliable and valid data, including

TABLE 1 | COVID-19 hospital length of stay.

\begin{tabular}{|c|c|c|c|c|c|}
\hline Study & Patients & Average & St. Deviation & Median & References \\
\hline 1 & 226 & 13 & NR & 4 & $(14)$ \\
\hline 2 & 144 & NR & NR & 13 & (15) \\
\hline 3 & 191 & NR & NR & 11 & $(16)$ \\
\hline 4 & 2,634 & $N R$ & NR & 4.1 & $(17)$ \\
\hline \multicolumn{3}{|c|}{ Patient weighted values } & & 4.91 & \\
\hline
\end{tabular}

Patients included in table were those that had discharge information reported. NR, Not Reported. 
data on the capacity and availability of hospital-based resources. Preferably, we would have widespread testing data to inform our epidemiological models and provide a leading indicator of future demands of our healthcare system. Ohio, and other states, were forced to prioritize testing due to lack of availability. The prioritization of limited COVID-19 tests based on CDC guidance emphasized healthcare workers first and those suspectible to the disease second, potentially increasing the spread of the novel coronavirus among those most at risk (18). The combination of widespread community transmission and lack of testing kits prevented us from having a clear understanding of the novel coronavirus spread, including those most at risk for requiring intensive care.

In the absence of wide spread testing prior to or at the initial onset of the epidemic, hospitalizations and hospital utilization become the second-best indicator to monitor the severity and progression of the novel coronavirus. Hospital utilization must be monitored to ensure that the hospitalization raw numbers do not become truncated. Once hospitals approach maximum capacity, the hospital's decision to triage and an individual's decision to seek care elsewhere or stay at home will introduce bias into the data measure. This necessity to avoid a biased indicator was the motivating reason to track hospitalizations in the first place. In geographic regions that are approaching capacity or where hospitals are already at maximum utilization, hospitalizations may be less indicative of COVID-19's prevalence. If this stage is reached however, any discussion about opening up hospitals for elective procedures is moot.

Using a novel data set from Ohio, this proposed framework provided a means to illustrate the monitoring and severity of the novel coronavirus while adjusting for daily fluctuations in the data. Our threshold of a declining 7-day moving average over a 14 -day period, "7\&14," provided a conservative threshold for informing public policy decisions, such as access to healthcare services, regarding the novel coronavirus pandemic.

Our approach is broadly consistent with the work of the University of Minnesota (UM), Carlson School of Management (19). The UM initiated a COVID-19 Hospitalization Tracking Project, and our work expands upon the efforts of UM by incorporating hospital capacity and providing a means to assess the ongoing epidemic. Baker et al. (20) proposed an approach for tracking influenza intensive care unit bed utilization to monitor severity of the influenza season (20). However, many states are not reporting hospitalizations reliably or at all, let alone intensive care beds to provide usual information that can be aggregated.

The proposed " $7 \& 14$ " framework has two key advantages. First, it can be implemented at the individual hospital level and aggregated by geographic regions, including other countries. It

\section{REFERENCES}

1. Sun K, Chen J, Viboud C. Early epidemiological analysis of the coronavirus disease 2019 outbreak based on crowdsourced data: a populationlevel observational study. Lancet Digital Heatlh. (2020) 2:e201-8. doi: 10.1016/S2589-7500(20)30026-1 requires three data inputs, hospitalizations, LOS, and occupancy. Second, one of the inherent benefits of using a moving average is to smooth out random short-term fluctuations in daily hospitalizations. These two attributes combined creates an easy to understand dashboard at the chosen level of analysis to assess the severity and spread of the novel coronavirus epidemic. If or when additional healthcare system supply data becomes available (i.e., intensive care bed utilization), this approach can easily be expanded.

The approach outlined presumes that a symptomatic patient presenting at the hospital will be admitted and diagnosed as probable or confirmed COVID-19 postive. Second, patients will remain hospitalized until they no longer require acute care services. If patients were to be discharged while still contagious, it may increase the rate of community spread requiring further hospital capacity. Similarly, if patients remain hospitalized until they are no longer contagious, there will be increased occupancy. In either case, the " $7 \& 14$ " approach outlined combining hospitalizations and utilizations can aid policy makers.

The proposed "7\&14" approach, leveraging hospitalizations and hospital utilization, may not be the ideal method of monitoring the novel coronavirus epidemic. The ideal method would include a robust testing and contact tracing strategy at the onset or prior to community transmission similar to South Korea or Singapore. However, widespread community transmission and lack of testing kits, elevates this approach to the best available. With improved reporting of COVID19 hospitalizations, LOS, and hospital occupancy across the country this approach may improve decision making for hospital administrators and policy makers.

\section{DATA AVAILABILITY STATEMENT}

The underlying data used in this analysis will be made available upon reasonable request.

\section{AUTHOR CONTRIBUTIONS}

All authors listed have made a substantial, direct and intellectual contribution to the work, and approved it for publication.

\section{ACKNOWLEDGMENTS}

The authors would like to thank Michael Topmiller, Ph.D., Edmond A. Hooker, MD, DrPH, Dee Ellingwood, MS, and Jennifer Mallow, MBA for their thoughtful comments and suggestions. Any errors or omissions are those of the authors alone.

2. Nsubuga P, White ME, Thacker SB, Anderson MA, Blount SB, Broome $\mathrm{CV}$, et al. Public health surveillance: a tool for targeting and monitoring interventions. In: Jamison DT, Breman JG, Measham AR, et al. editors. Disease Control Priorities in Developing Countries. 2nd edition. New York, NY: The International Bank for Reconstruction and Development/The World Bank, Oxford University Press. Chapter 53 (2006). Available 
online at: https://www.ncbi.nlm.nih.gov/books/NBK11770/ (accessed June 25, 2020).

3. Hashimoto S, Murakami Y, Taniguchi K, Nagai M. Detection of epidemics in their early stage through infectious disease surveillance. Int J Epidemiol. (2000) 29:905-10. doi: 10.1093/ije/29.5.905

4. Gandhi M, Yokoe DS, Havlir DV. Aysmptomatic transmission, the achilles' heel of current strategies to control COVID-19. NEng J Med. (2020) 382:215860. doi: 10.1056/NEJMe2009758

5. Ing AJ, Cocks C, Green JP. COVID-19: in the footsteps of Ernest Shackleton. BMJ Thorax. (2020). doi: 10.1136/thoraxjnl-2020-215091

6. Ohio Department of Health (ODH). COVID-19 Presser Final. (2020) Available online at: https://www.coronavirus.ohio.gov/static/slides/04-22-20-PresserFinal.pdf (accessed on June 25, 2020).

7. The Centers for Disease Control and Prevention (CDC). Coronavirus. (2020). Available online at: www.cdc.gov/coronavirus (accessed April 19, 2020).

8. The Centers for Disesae Control and Prevention (CDC). National Center for Health Statistics. Occupancy Rates in Community Hospitals. (2016). Available online at: https://www.cdc.gov/nchs/data/hus/2017/091.pdf (accessed on April 19, 2020).

9. Definitive Healthcare. COVID-19 Capacity Predictor. Available online at: www.definitivehc.com/resources/covid-19-capacity-predictor (accessed April 20, 2020).

10. Buckingham-Jeffery E, Morbey R, House T, Elliot AJ, Harcourt S, Smith GE. Correcting for day of the week and public holiday effects: improving a national daily syndromic surveillance service for detecting public health threats. $B M C$ Public Health. (2017) 17:477. doi: 10.1186/s12889-017-4372-y

11. Ng Y, Li Z, Chua YX, Chaw WL, Zhao Z, Er B, et al. Evaluation of the effectiveness of surveillance and containment measures for the first 100 patients with COVID-19 in Singapore - January 2-February 29, 2020. Morb Mortal Wkly Rep. (2020) 69:307-11. doi: 10.15585/mmwr.mm 6911e1

12. Rothman KJ, Greenland S, Lash TL. Modern Epidemology. Philadelphia, PA: Lippincott Williams and Wilkins (2008).

13. Lauer SA, Grantz KH, Bi Q, Jones FK, Zheng Q, Meredith HR, et al. The incubation period of coronavirus disease 2019 (COVID-19) from publicly reported confirmed cases: estimation and application. Ann Internal Med. (2020) 172:577-82. doi: 10.7326/M20-0504
14. Kozak R, Prost K, Yip L, Villiams V, Leis JA, Mubareka S. Severity of coronavirus respiratory tract infections in adults admitted to acute care in Toronto, Ontario. J Clin Virol. (2020) 120:104338. doi: 10.1016/j.jcv.2020.104338

15. Wu C, Xiaoyan C, Cai Y, Xia J, Zhou X, Xu S, et al. Risk factors associated with acute respiratory distress syndrome and death in patients with coronavirus disease 2019 Pneumonia in Wuhan, China. JAMA Intern Med. (2020) 13:e200994. doi: 10.1001/jamainternmed.2020.0994

16. Zhou F, Yu T, Du R, Fan G, Liu Y, Liu Z, et al. Clinical course and risk factors for mortality of adult inpatients with COVID-19 in Wuhan, China: a retrospective cohort study. Lancet. (2020) 395:P1065-2. doi: 10.1016/S0140-6736(20)30566-3

17. Richardson S, Hirsch JS, Narasimhan M, Crawford JM, McGinn T, Davidson KW, et al. Presenting Characteristics, comorbidities, and outcomes among 5700 patients hospitalized with COVID-19 in the New York City Area. JAMA. (2020) 323:2052-9. doi: 10.1001/jama.2020.6775

18. Gurwitz JH. COVID-19, post-acute care preparedness and nursing homes: flawed policy in the fog of war. J Amer Geriatrics Soc. (2020) 68:1124-5. doi: 10.1111/jgs.16499

19. Health Affairs. Calling All States to Report Standardized Information on COVID-19 Hospitalizations. (2020). Health Affairs Blog. Available online at: https://www.healthaffairs.org/do/10.1377/hblog20200406.532030/ full/ (accessed June 25, 2020).

20. Baker AW, Edmond MB, Herwaldt LA, Chen LF, Srikantaswamy S, Sexton DJ. Real-time surveillance of inluenza morbidty: tracking intensive care unit resource utilization. Ann Am Thoracic Soc. (2017) 14:1810-7. doi: 10.1513/AnnalsATS.201609-721OC

Conflict of Interest: The authors declare that the research was conducted in the absence of any commercial or financial relationships that could be construed as a potential conflict of interest.

Copyright (c) 2020 Mallow and Jones. This is an open-access article distributed under the terms of the Creative Commons Attribution License (CC BY). The use, distribution or reproduction in other forums is permitted, provided the original author(s) and the copyright owner(s) are credited and that the original publication in this journal is cited, in accordance with accepted academic practice. No use, distribution or reproduction is permitted which does not comply with these terms. 